\title{
A potential therapeutic strategy for chronic Iymphocytic leukemia by combining Idelalisib and GS-9973, a novel spleen tyrosine kinase (Syk) inhibitor
}

\author{
Russell T Burke ${ }^{1}$, Sarah Meadows ${ }^{2}$, Marc M Loriaux ${ }^{1,4}$, Kevin S. Currie ${ }^{3}$, Scott \\ A. Mitchell ${ }^{3}$, Patricia Maciejewski ${ }^{3}$, Astrid S. Clarke ${ }^{3}$, Julie A. Dipaolo ${ }^{3}$, Brian J. \\ Druker ${ }^{1,5}$, Brian J. Lannutti ${ }^{2}$, and Stephen E. Spurgeon ${ }^{1,6}$ \\ ${ }^{1}$ Knight Cancer Institute, Oregon Health \& Science University, Portland, OR. \\ ${ }^{2}$ Gilead Sciences Inc, Seattle, WA \\ ${ }^{3}$ Gilead Sciences Inc, Branford, CT \\ ${ }^{4}$ Division of Pathology, Oregon Health \& Science University, Portland, OR \\ ${ }^{5}$ Howard Hughes Medical Institute, Bethesda, MD \\ ${ }^{6}$ Division of Hematology and Medical Oncology, Oregon Health \& Science University \\ Correspondence to: Stephen E. Spurgeon, email: spurgeos@ohsu.edu \\ Keywords: Chronic Lymphocytic Leukemia, B-cell receptor, signaling pathways, spleen tyrosine kinase, PI3 kinase \\ Received: October 9, $2013 \quad$ Accepted: October 28, $2013 \quad$ Published: October 30, 2013
}

This is an open-access article distributed under the terms of the Creative Commons Attribution License, which permits unrestricted use, distribution, and reproduction in any medium, provided the original author and source are credited.

ABSTRACT:

Agents that target B-cell receptor (BCR) signaling in lymphoid malignancies including idelalisib (GS-1101) and fostamatinib which inhibit the delta isoform of PI3 kinase (PI3Kd) and spleen tyrosine kinase (Syk) respectively have shown significant clinical activity. By disrupting B-cell signaling pathways, idelalisib treatment has been associated with a dramatic lymph node response, but eradication of disease and relapse in high risk disease remain challenges. Targeting the BCR signaling pathway with simultaneous inhibition of PI3Kd and Syk has not yet been reported. We evaluated the pre-clinical activity of idelalisib combined with the novel and selective Syk inhibitor GS-9973 in primary peripheral blood and bone marrow Chronic Lymphocytic Leukemia (CLL) samples. Both PI3Kd and Syk inhibition reduced CLL survival and in combination induced synergistic growth inhibition and further disrupted chemokine signaling at nanomolar concentrations including in bone marrow derived and poor risk samples. Simultaneous targeting of these kinases may significantly increase clinical activity.

\section{INTRODUCTION}

Chronic lymphocytic leukemia (CLL) remains incurable with standard therapies [1]. Increasingly it has been recognized that CLL cell survival is dependent on the complex interactions of neoplastic cells with the microenvironment. Specifically, cell proliferation is mediated by multiple inputs such as B-cell receptor (BCR) signaling pathways, chemokines, cytokines, integrins, nurse-like cells, BAFF, and CD40 [2]. A number of therapeutic agents have been developed including small molecules that inhibit these key signaling pathways including agents that target BCRmediated kinase signaling pathways and disrupt CLL cell-microenvironment interactions $[3,4]$. Treatment of primary CLL cells with idelalisib (GS-1101), ibrutinib, and fostamatinib (R406) which inhibit the PI3 kinase delta-specific isoform (PI3Kd), Bruton's tyrosine kinase (Btk) and spleen tyrosine kinase (Syk) respectively, results in inhibition of BCR signaling pathways, decreased cell proliferation, and disruption of chemokine mediated CLL cell migration [5],[6],[7],[8]. These agents are orally bioavailable and have been evaluated in early phase trials in relapsed and refractory CLL patients. Although significant clinical activity has been observed in patients treated with these drugs as single agents, complete remission rates are low and marrow disease may be difficult to eradicate. Furthermore, although response 
rates with monotherapy do not appear to be adversely affected by the presence of poor risk disease, responses are significantly less durable in this population [9, Brown J.R. et al. J Clin Oncolo 31, 2013 (supple;abstr 7003)]. Thus new therapeutic approaches that evaluate these agents in combination are warranted.

Currently, little is known about the effects of inhibiting multiple nodes in the BCR pathway. Feedback loops and cross talk between signaling pathways may significantly impact the efficacy of cancer therapeutics and drive resistance to single agent therapy. Combination therapy to address the molecular complexity associated with the convergence of B-Cell signaling pathways could provide a novel treatment approach. Inhibition of multiple B-cell signaling pathways and simultaneous inhibition of the BCR signaling pathway may have the potential for synergy and implications for overcoming resistance to single agents or eradicating minimal residual disease (MRD) the latter of which has been shown to correlate with survival after chemo-immunotherapy [10]. These considerations prompted us to assess the effects of dual $\mathrm{PI} 3 \mathrm{~K} \delta$ and Syk inhibition in CLL using idelalisib and the novel Syk inhibitor GS-9973.

\section{RESULTS}

\section{The Combination of Idelalisib and GS-9973 Synergistically Inhibits Cell Viability at Nanomolar Concentrations in vitro}

Significant synergy was seen in the majority of samples treated with idelalisib and GS-9973. A heat map (Figure 1A) of plotted interaction indices depicting the sensitivity to the combination is shown. For the majority of samples without synergistic responses, additive interactions were observed. Specific disease and/or biologic characteristics are shown in Table 1. Half of all samples were obtained from patients with relapsed disease and two of four bone marrow derived samples were from patients with refractory disease. Three samples harbored a 17p deletion and seven samples had an unmutated variable region of the immunoglobulin heavy
A
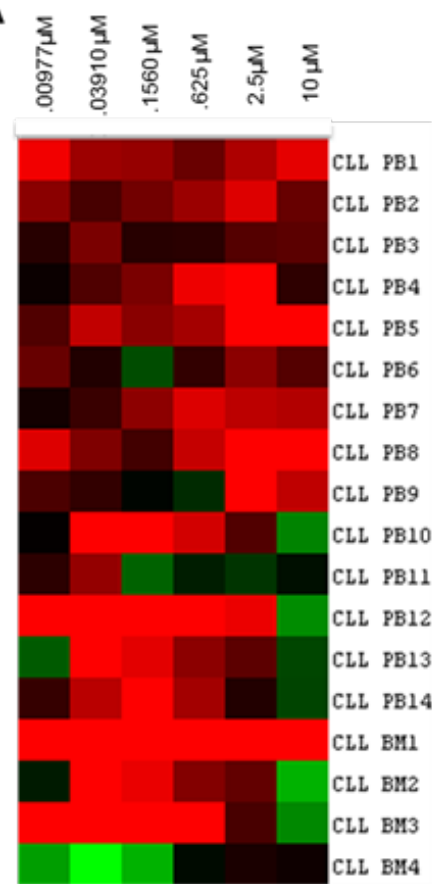

B

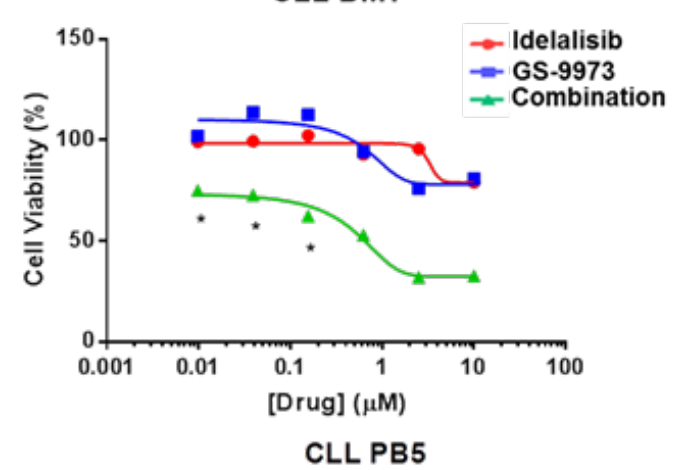

antagonistic

additive

synergistic

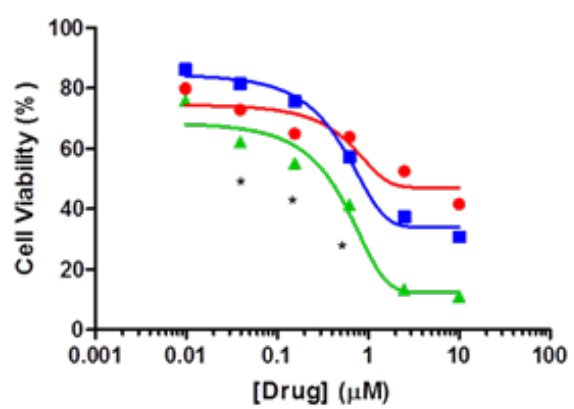

Figure 1: (A) A heat map displaying interaction indices at individual concentration points for each CLL sample. $\mathrm{R}$ values $>1$ (antagonistic), 1 (additive) and $<1$ (synergistic) are color coded as green, black and red, respectively. Specifically, the heat map was created using TreeView by transforming the interaction index for each concentration point of the primary samples by $\log _{2}$. Each row represents an individual sample. Peripheral blood samples are labeled "PB" and bone marrow samples are labeled "BM." The columns represent (from left to right) increasing equimolar concentrations of each drug drug ranging from $.977 \mathrm{nM}$ to $10 \mu \mathrm{M}$. (B) A representative viability curve for CLL BM1 is shown with viability on the $\mathrm{y}$ axis and increasing equimolar concentrations of drug $(\mu \mathrm{M})$ on the $\mathrm{x}$ axis. Resistance to each agent as monotherapy is seen (top 2 curves), an effect that is overcome by the drug combination (bottom curve)when the combination is used. (C)) Viability curve for a sample representative of having synergy. Although all concentrations of the drug combination were synergistic, (CI $<$ 1), some concentrations had values that lost significance when accounting for the $95 \%$ confidence interval. Therefore, asterisks are provided which denote points that are significantly synergistic within the $95 \%$ confidence interval as defined by R. Idelalisib (GS-1101) treated cells are shown in red, GS-9973 in blue, and the combination in green. 
Table 1: Disease status and biologic CLL disease characteristics.

\begin{tabular}{|l|l|l|l|l|l|}
\hline $\begin{array}{l}\text { Sample } \\
\text { ID }\end{array}$ & $\begin{array}{l}\text { Disease } \\
\text { Status }\end{array}$ & $\begin{array}{l}\text { Cytogenetics/ } \\
\text { FISH }\end{array}$ & $\begin{array}{l}\text { IgVh mutational } \\
\text { status }\end{array}$ & ZAP 70 & CD38 \\
\hline PB 1 & Relapsed & Normal & Mutated & Negative & Negative \\
\hline PB 2 & Untreated & Normal & Mutated & Negative & Negative \\
\hline PB 3 & Relapsed & Unknown & Unknown & Unknown & Unknown \\
\hline PB 4 & Relapsed & $\begin{array}{l}17 p \text { deletion } \\
13 q \text { deletion }\end{array}$ & Unmutated & Positive & Positive \\
\hline PB 5 & Untreated & $13 q$ deletion & Mutated & Negative & Negative \\
\hline PB 6 & Untreated & $13 q$ deletion & Mutated & Unknown & Unknown \\
\hline PB 7 & Untreated & $13 q$ deletion & Mutated & Negative & Negative \\
\hline PB 8 & Untreated & $13 q$ deletion & Mutated & Negative & Negative \\
\hline PB 9 & Relapsed & $13 q$ deletion & Unknown & Unknown & Unknown \\
\hline PB 10 & Relapsed & $\begin{array}{l}11 q \text { deletion } \\
13 q \text { deletion }\end{array}$ & Unmutated & Negative & Positive \\
\hline PB 11 & Untreated & $13 q$ deletion & Mutated & Unknown & Unknown \\
\hline PB 12 & Relapsed & Trisomy 12 & Unmutated & Positive & Negative \\
\hline PB 13 & Unknown & Unknown & Unknown & Unknown & Unknown \\
\hline PB 14 & Refractory & $\begin{array}{l}\text { Complex } \\
17 p \text { deletion } \\
\text { Trisomy 12 }\end{array}$ & Unmutated & Positive & Negative \\
\hline BM 1 & Refractory & $13 q$ deletion & Unmutated & Positive & Positive \\
\hline BM 2 & Relapsed & $\begin{array}{l}13 q \text { deletion } \\
\text { Trisomy 12 }\end{array}$ & Umutated & Positive & Negative \\
\hline BM 3 & Refractory & $\begin{array}{l}17 p \text { deletion } \\
13 q \text { deletion }\end{array}$ & Unknown & Negative & Negative \\
\hline
\end{tabular}

chain (IgVh). Disease factors, such as relapsed/refractory disease, IgVh mutational status, and fluourescent in situ hybridization results (FISH) did not correlate with achieving a synergistic response. However, notably, of the 3 samples (PB4, PB14, and BM3) harboring a17p deletion synergy was seen. Representative cell viability curves for individual samples are shown (Figure 1B and 1C). Data for all single agent and combination viability curves are also included (Supplemental Figure 1). Three of four bone marrow derived samples showed synergy. Interestingly, CLL BM1 was resistant (i.e. no significant decrease in cell viability) to each drug alone but synergistically sensitive to the combination.

\section{Idelalisib and GS-9973 Inhibit BCR mediated signaling pathways}

To confirm that idelalisib and GS-9973 effectively inhibit BCR mediated signaling, we evaluated phosphorylation of Akt and ribosomal S6 in CLL cells after treatment with idelalisib and GS-9973. Treatment with each drug alone significantly decreased pAkt at nanomolar concentrations while combination treatment did not result in a significant further decline in pAkt when compared to each inhibitor alone. Conversely, although single agent treatment with idelalisib and GS9973 decreased S6 phosphorylation, the combination was significantly more potent (Figure 2B). To further evaluate these agents ability to inhibit BCR mediated signaling after IgM stiumulation, using immunoblotting, we also evaluated the inhibitory effects on Ramos cells, a Burkitt's cell line with an intact BCR. A significant decrease in AKT phosphorylation was seen with each drug alone while ERK phosphorylation was inhibited by GS-9973 +/- GS-1101. S-6 phosphorylation was also inhibited by the combination (data not shown).

\section{Effects of Combination of Chemokine/Cytokine Networks}

When CLL cells were co-cultured with HS5 stromal cells $(\mathrm{n}=6)$, significantly higher levels of CCL2, CCL3, CCL4 and CCL22 were seen (Figure 2). Combination treatment significantly decreased CCL2 (2C), CCL3 (2D), CCL4 (2E), CCL22 (2F) compared to each individual drug. To validate the results seen using the HS-5 coculture system, we also tested the effects of idelalisib or GS-9973 on CLL cells cultured in primary BM stromal cells $(n=3)$ and saw significant decreases in chemokine levels (Figure 2G) after idelalisib plus GS-9973 treatment. 
A

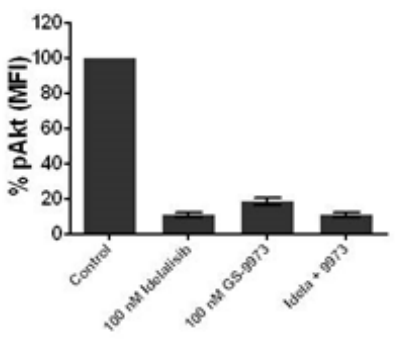

D

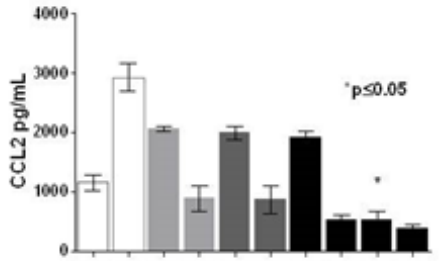

\begin{tabular}{l|l|l|l|l|l|l|l|l|l|l|}
\hline HS- 5 & - & + & + & + & + & + & + & + & + & + \\
\hline
\end{tabular}

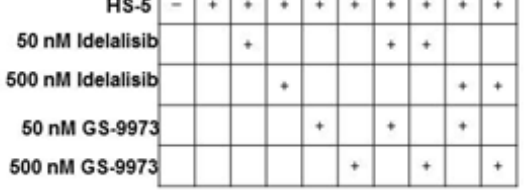

E
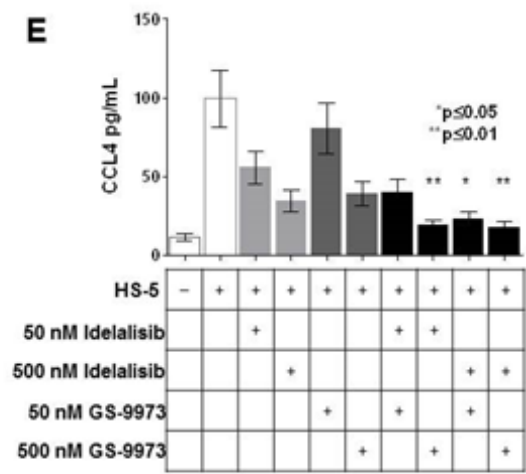

B
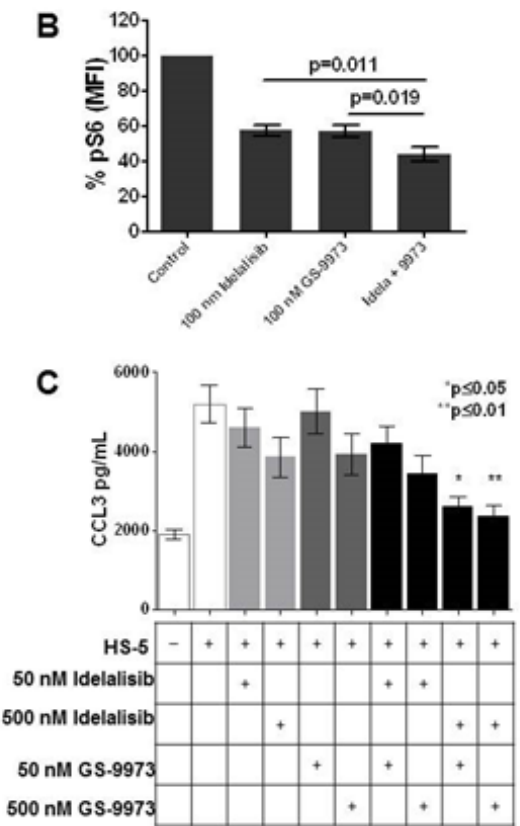

$\mathbf{F}$

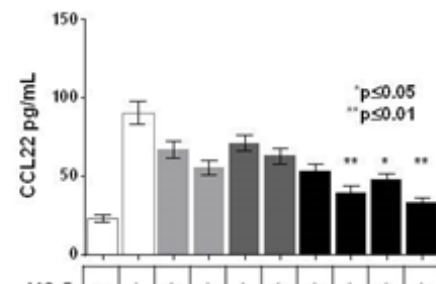

\begin{tabular}{ll|l|l|l|l|l|l|l|l|l|}
\hline HS-5 & - & + & + & + & + & + & + & + & + & + \\
\hline
\end{tabular}

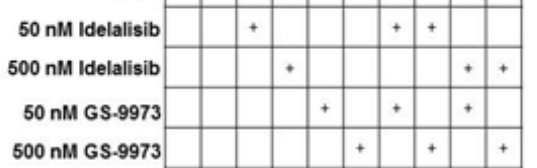

G
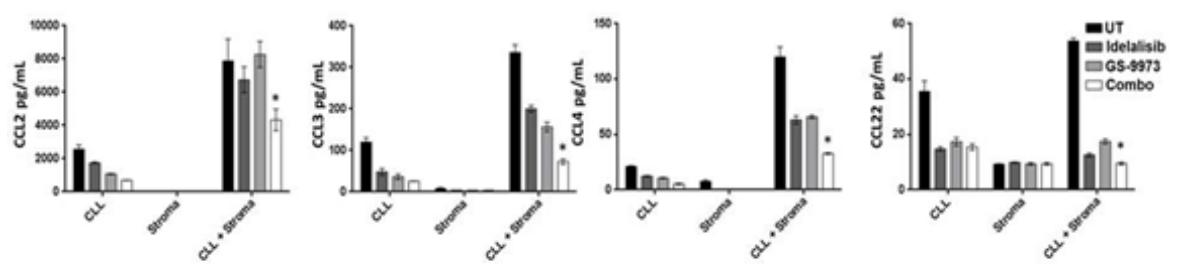

Figure 2: Treatment of primary CLL cells $(n=14)$ co-cultured with HS5 stromal cells with idelalisib $(100 \mathrm{nM})$ or GS-9973 (100 nM), alone or in combination (100 nM each), results in decreased AKT phosphorylation. (A) while treatment with the combination significantly decreases S6 phosphorylation as compared to treatment with each drug alone $(\mathrm{p}=0.011$ for the combination compared to GS-1101 treatment alone and $\mathrm{p}=0.019$ compared to GS-9973 alone). Each condition was compared using a two tailed t-test. (B)The effect of idelalisib (GS-1101) and/or GS-9973 on CCL2, CCL3, CCL4, and CCL22 expression after CLL-HS-5 co-culture. The bar diagrams represent the mean supernatant concentrations (+/- SEM) of CCL2 (C), CCL3 (D), CCL4 (E), CCL22 (F) from CLL cells co-cultured with or without (controls) HS-5 cells and various concentrations of idelalisib, GS-9973, or a combination of idelalisib and/GS-9973 (50 nM plus $50 \mathrm{nM}, 50 \mathrm{nM}$ plus $500 \mathrm{nM}$ for each drug, and $500 \mathrm{nM}$ plus $500 \mathrm{nM}$ for each drug) in 6 different patient samples assessed after 24 hours. Chemokine expression after treatment with individual drug or the combination was compared using a two tailed t-test. The combination of idelalisib and GS-9973 showed significant effect on decreasing CCL2, CCL3, CCL4, CCL22. A single asterisk denotes significant $(\mathrm{p}<.05)$ and a double asterisk $(* *)$ represents a significance of $\mathrm{p}<.01$. Differences between single agent treatment and the combinations are shown. Panels G-J show increased chemokine expression when CLL cells are cultured in the presence of primary bone marrow derived stromal cells $(\mathrm{n}=3)$. Similar to the HS5 co-culture system, co-culture with primary bone marrow leads to increased chemokine expression (mean +/- SEM supernatant concentrations) which is abrogated by treatment with idelalisib (100 nM) and GS-9973( $100 \mathrm{nM}$ ) for 24 hours. As shown in the last column in each figure, this effect that is significantly enhanced by combining these two agents $(\mathrm{p}<.05$, two tailed $\mathrm{t}$-test). 
Table 2: Chemical characterization of GS-9973. Panel A shows the chemical structure for GS-9973. Panel B shows the relative selectivity of GS-9973 compared to the Syk inhitor R406 using the DiscoveRx panel for Kd determinations

\begin{tabular}{|c|c|c|c|c|c|c|c|c|c|}
\hline \multirow[b]{2}{*}{$\begin{array}{c}\text { KnomeScan } \\
\text { Symbol }\end{array}$} & \multicolumn{2}{|c|}{ GS-9973 } & \multicolumn{2}{|c|}{ R406 } & \multirow{2}{*}{$\begin{array}{c}\text { KinomeScan } \\
\text { Symbol }\end{array}$} & \multicolumn{2}{|c|}{ GS-9973 } & \multicolumn{2}{|c|}{ R406 } \\
\hline & $\begin{array}{c}\text { Kd } \\
(n M)\end{array}$ & $\begin{array}{l}\text { Selectis ty } \\
\text { (Fold) }\end{array}$ & $\begin{array}{c}\text { Kd } \\
(\mathrm{nM})\end{array}$ & $\begin{array}{l}\text { Selectivity } \\
\text { (Fold) }\end{array}$ & & $\begin{array}{c}\mathrm{Kd} \\
(\mathrm{nM})\end{array}$ & $\begin{array}{l}\text { Selectiv ty } \\
\text { (Fold) }\end{array}$ & $\begin{array}{c}\mathrm{Kd} \\
(\mathrm{nM})\end{array}$ & $\begin{array}{l}\text { Selectivity } \\
\text { (Fold) }\end{array}$ \\
\hline ABL1-nonphos. & 8400 & 800 & 8400 & 560 & LTK & 1200 & 114 & 800 & 53 \\
\hline ABL1-phos. & 560 & 53 & 560 & 37 & MAP4K2 & 40000 & 3810 & 670 & 45 \\
\hline ALK & 2900 & 276 & 240 & 16 & MEK5 & 200 & 19 & 27 & 1.8 \\
\hline $\mathrm{AXX}$ & 1900 & 181 & 82 & 5 & MERTK & 3300 & 314 & 170 & 11 \\
\hline BIKE & 1100 & 105 & 1900 & 127 & MLK1 & 570 & 54 & 4.3 & 0.3 \\
\hline BLK & 420 & 40 & 25 & 1.7 & PDGFRB & 670 & 64 & 3.3 & 0.2 \\
\hline BRK & 330 & 31 & 360 & 24 & PIP5K1A & 40000 & 3810 & 1900 & 127 \\
\hline CHEK2 & 40000 & 3810 & 1000 & 67 & PIP5K1C & 40000 & 3810 & & \\
\hline CSF1R & 180 & 17 & 31 & 2.1 & PIP5K2B & 40000 & 3810 & 1200 & 80 \\
\hline CSNK2A1 & 540 & 51 & 1400 & 93 & PRKR & 3000 & 286 & 180 & 12 \\
\hline CSNK2A.2 & 110 & 10 & 1200 & 80 & RET & 1000 & 95 & 4.1 & 0.3 \\
\hline DAPK1 & 40000 & 3810 & 55 & 4 & RIOK1 & 40000 & 3810 & 340 & 23 \\
\hline DAPKB & 40000 & 3810 & 13 & 0.9 & RIOK2 & 160 & 15 & 3400 & 227 \\
\hline EGFR & 1400 & 133 & 300 & 20 & RIOK3 & 40000 & 3810 & 210 & 14 \\
\hline EPHA1 & 470 & 45 & 67 & 4 & SBK1 & 40000 & 3810 & 93 & 6 \\
\hline EPHB6 & 550 & 52 & 310 & 21 & SLK & 820 & 78 & 33 & 2.2 \\
\hline FGR & 230 & 22 & 33 & 2.2 & SRC & 110 & 10 & 8.4 & 0.6 \\
\hline FLT3 & 320 & 30 & 0.71 & 0.05 & SRMS & 910 & 87 & 610 & 41 \\
\hline FLT3-autoinhib. & 40000 & 3810 & 13 & 0.9 & SRPK1 & 40000 & 3810 & 590 & 39 \\
\hline GAK & 1300 & 124 & 250 & 17 & SRPK3 & 3200 & 305 & 400 & 27 \\
\hline GRK 4 & 40000 & 3810 & 450 & 30 & STK16 & 330 & 31 & 1.7 & 0.1 \\
\hline $\mathrm{HCK}$ & 410 & 39 & 150 & 10 & STK33 & 40000 & 3810 & 970 & 65 \\
\hline HIPK1 & 40000 & 3810 & 820 & 55 & SYK & 10.5 & 1 & 15 & 1 \\
\hline HIPK3 & 40000 & 3810 & 3200 & 213 & TAK1 & 490 & 47 & 49 & 3 \\
\hline INSR & 1800 & 171 & 1100 & 73 & TGFBR2 & 390 & 37 & 330 & 22 \\
\hline $\begin{array}{l}\text { IRAK1 } \\
\text { IRAK3 }\end{array}$ & 140 & 13 & 9.7 & $\begin{array}{l}0.6 \\
0.7\end{array}$ & TN K1 & 86 & 8 & 25 & 1.7 \\
\hline $\begin{array}{l}\text { IRAK3 } \\
\text { JAK1(JH1-cat.) }\end{array}$ & $\begin{array}{l}120 \\
730\end{array}$ & $\begin{array}{l}11 \\
70\end{array}$ & $\begin{array}{l}11 \\
21\end{array}$ & $\begin{array}{l}0 . \\
1.4\end{array}$ & TNK2 & 670 & 64 & 70 & 5 \\
\hline JAK 2(JH1-cat.) & 110 & 10 & 3.5 & 0.2 & TYK2(JH1-cat.) & 180 & 17 & 190 & 13 \\
\hline JAK3(JH1-cat.) & 3200 & 305 & 36 & 2.4 & ULK3 & 510 & 49 & 7.1 & 0.5 \\
\hline JNK1 & 2000 & 190 & 38 & 3 & VEGF R2 & 40000 & 3810 & 40 & 3 \\
\hline KIT & 210 & 20 & 6.8 & 0.5 & YES & 450 & 43 & 35 & 2.3 \\
\hline LCK & 820 & 78 & 30 & 2.0 & YSK4 & 440 & 42 & 67 & 4 \\
\hline LOK & 40000 & 3810 & 70 & 5 & ZAP70 & 410 & 39 & 21 & 1.4 \\
\hline
\end{tabular}

\section{DISCUSSION}

Here we show that the combination of idelalisib and GS-9973 synergistically decreases CLL cell viability in the majority of CLL samples tested. Significant growth inhibition was seen in the presence of HS- 5 conditioned media and in patient derived primary bone marrow CLL cells, which are known to have an activated phenotype [13] and be more resistant to cytotoxic therapy [14], [15]. Interestingly one bone marrow derived sample that was resistant to each drug as a single agent demonstrated significant growth inhibition when treated with idelalisib and GS-9973 in combination. This suggests that this difficult to eradicate disease compartment may potentially be successfully targeted with simultaneous PI3Kd and Syk inhibition. This may not only enhance treatment efficacy but also suggests a role for treating minimal residual disease, the presence of which is associated with treatment failure after initial therapy [16].

As drugs that target the $\mathrm{BCR}$ are not classic cytotoxic agents, their ability to inhibit their downstream targets and disrupt chemokine/cytokine interactions, which are essential for CLL cell homing and survival, are critical 
to their mechanism of action. Akt and S6 are known to be regulated by PI3K and Syk which are effected by BCR activation [17],[18]. PI3K also receives co-stimulatory signals from BAFF, CD40, and toll-like receptor pathways while Fc receptors, chemokines, and integrins can co-stimulate Syk activation [2]. Phospho-Akt (pAkt) has previously been shown to decrease after idelalisib treatment [19] and with Syk inhibition [20]. Here we confirm that treatment of primary CLL cells with idelalisib decreases pAkt. GS-9973 also inhibits pAkt at nanomolar concentrations. Combination treatment did not result in a significant further decline in pAkt when compared to each inhibitor alone. The finding that the combination's ability to further inhibit S6 phosphorylation is not surprising as S6 lies downstream of multiple kinase pathways and again highlights the potential efficacy of inhibiting two tightly integrated yet distinct signaling pathways.

Chemokine and cytokine levels have been shown to be elevated in CLL and are known to be an integral part in maintenance of the CLL cell microenvironment interaction [21]. Increased expression of CCL3 and CCL4 has been seen in CLL after BCR activation or coculture with nurse like cells (NLCs) and may contribute to recruitment of T-cells [22]. Recruitment of T-cells is further augmented by CCL22 production while CCL2 has been shown to augment CLL cell survival in vitro [23]. In addition, stromal cell chemokines, such as CXCL12, aid in CLL chemotaxis into the stromal compartment and activation of pro-survival signaling. This effect has been shown to be abrogated by treatment with a number of agents that target BCR mediated pathways including Syk [25]. In addition, it is well established that patients treated with idelalisib have significant reductions in CCL3 and CCL4 and disruption of these networks results in significant migration of CLL cells out of lymph nodes. Notably, we found that when CLL cells were cultured with HS-5 stromal cells or primary bone marrow cells, increases in CCL2, CCL3, CCL4, and CCL22 were seen. Interestingly, after treatment with each drug alone, inhibition of chemokine expression was observed an effect that was significantly enhanced when using idelalisib and GS-9973 in combination at $\mathrm{nM}$ concentrations. These data suggest that using this combination can further disrupt chemokine networks known to be essential to CLL homing and survival. These findings may have implications for treating resistance to chemo-immunotherapy, single agent kinase inhibitors, or for targeting minimal residual disease especially in poor risk patients who have significantly shorter remissions with monotherapy.

We have shown that the combination of idelalisib and the novel Syk inhibitor, GS-9973, is effective at synergistically decreasing CLL cell viability, inhibiting BCR mediated signaling, and disrupting chemokine expression in CLL. These data provide rationale for the clinical combination of agents including PI3Kd and Syk inhibitors in CLL that target multiple B-cell signaling pathways and simultaneously target BCR mediated pathways. Such Informed combinations, directed at bypassing feedback loops and interrupting cross talk between signaling pathways may improve therapeutic outcomes and continue to significantly inform our approach to CLL treatment.

\section{MATERIALS AND METHODS}

\section{Cell isolation and culture}

After informed consent, in accordance with the Declaration of Helsinki and approved by the institutional review board, lymphocytes were isolated from the peripheral blood $(n=14)$ or bone marrow $(n=4)$ of CLL patients and purified using a Ficoll-Paque PLUS (GE Healthcare) gradient. Disease status and biologic characteristics for each sample was recorded. Cells were used fresh or collected for viable frozen samples in fetal bovine serum with 10\% DMSO and stored in liquid nitrogen. Culture media was made with RPMI-1640 (Gibco) supplemented with 10\% fetal bovine serum, $4 \mathrm{mM}$ L-Glutamine, penicillin and streptomycin. Conditioned media was prepared by culturing the RPMI over confluent HS-5 human stromal cell line for 3 hours.

\section{Inhibitor combinations and synergy}

Ficoll purified CLL cells $\left(2 \times 10^{5}\right)$ isolated from bone marrow or peripheral blood were treated with each drug alone and with six equimolar concentrations of idelalisib and GS-9973-obtained from Gilead Sciences, Inc. ranging from $.977 \mathrm{nM}$ to $10 \mu \mathrm{M}$ on 96 -well plates in triplicate. GS9973 is highly selective for Syk. Its chemical structure and kinase selectivity using the DiscoveRx kinome panel (San Diego, CA) for Kd determinations as compared to R406 are shown in Table 2. Plated cells were then cultured in HS- 5 conditioned media at $37^{\circ} \mathrm{C}$ with $5 \% \mathrm{CO}_{2}$. After 72 hours of culture, viability was determined using an MTS assay (Cell Titer 96, Promega). Viability data were used to generate cell viability curves for each drug alone and in combination for each sample. The potential synergy of the combination of idelalisib and GS-9973 at a given equimolar concentration was determined using the median effect model [11]. The statistical modeling was run in $\mathrm{R}$ using a script that utilizes the median effect model [12]. A value of 1 , less than 1 , and greater than 1 using $R$ defines an additive interaction, synergistic and antagonistic, respectively. The Lee et al. method calculates a 95\% confidence interval for each data point. For each viability curve, to be considered synergistic, a data point must have an interaction index below 1 and the upper confidence interval must also be below 1 . In order to summarize and demonstrate collective synergy results, a heat map was 
created using TreeView by transforming the interaction index for each concentration point of the primary samples by $\log _{2}$.

\section{Inhibition of BCR mediated signaling pathways}

For flow cytometry experiments, ficoll purified CLL cells obtained from 14 patients were co-cultured with HS5 stromal cells and then treated with each drug alone $(100 \mathrm{nM})$ or in combination (100 $\mathrm{nM}$ each) for 24 hours. CLL cells were stained with anti-CD5fluorescein isothiocyanate (FITC) (Clone BL1a, Beckman Coulter, Indianapolis IN) and anti-CD19- Phycoerythrin (PE) (Clone 1D3, BD Biosciences, San Jose CA) to identify CLL cells, and either anti- phopho-Akt ${ }^{\mathrm{S} 473}$, anti-phospho-S6 (Alexa Fluor 488, Life Technologies, Carlsbad, CA) or an isotype-matched control antibody (mouse IgG1-Alexa Fluor 488 conjugate, Cell Signaling, Danvers, MA). FITC-CD5/CD19 ${ }^{+}$cells were gated (using anti-CD5-FITC and Anti-CD19-PE) and analyzed by 3-color flow cytometry to quantify intracellular phospho- $\mathrm{Akt}^{\mathrm{S} 473}$ levels (Cytomics FC 500MPL cell sorter and MXP Version 2.2 software, Beckman Coulter, Brea, CA).

To further validate these agents' ability to inhibit BCR mediated signaling we evaluated Ramos cells, a Burkitt's cell line with intact BCR. $5 \times 10^{6}$ cells were plated and treated with $1 \mu \mathrm{M}$ of idelalisib, GS-9973 alone or in combination and were cultured for 24 hours in standard

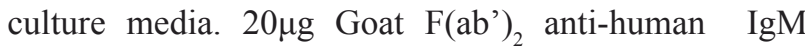
(Invitrogen) was added to stimulate the BCR for $30 \mathrm{~min}$. Protein isolates were subjected to immune-blotting with Phospho-Akt (S473), phospho-Erk (T202/Y204) (Cell Signaling), and phospho-S6 (Cell signaling).

\section{Chemokine expression in HS-5 co-culture or primary bone marrow}

The effect of idelalisib and/ or GS-9973 treatment on chemokine expression after CLL- HS-5 co-culture $(n=6)$ or CLL co-culture with primary bone marrow derived CLL cells $(n=3)$ was measured after 24 hours of incubation with individual drug $(50 \mathrm{nM}$ or $500 \mathrm{nM})$ or in combination using four conditions (50 nM plus 50 $\mathrm{nM}, 50 \mathrm{nM}$ plus $500 \mathrm{nM}$ for each drug, and $500 \mathrm{nM}$ plus $500 \mathrm{nM}$ for each drug). For the marrow samples, ficoll purified frozen CLL samples from the same patient were thawed and $5 \times 10^{6}$ cells added to marrow stromal cells and incubated with each drug alone or in combination in triplicate. Supernatants were harvested and assayed for CCL2, CLL3, CCL4, and CCL22 by quantitative ELISA (Quantikine; R\&D Systems). A two tailed t-test was used to compare the effect of drug combinations compared to treatment with each individual drug.

\section{AUTHORSHIP}

RTB designed research, performed research, analyzed data, and wrote the paper, $S M$ performed research, analyzed data, and wrote the paper, $M M L$ designed research and wrote the paper, $K S C$ performed research and wrote the paper, $S A M$ performed research and wrote the paper, $P M$ performed research and wrote the paper, $A S C$ performed research and wrote the paper, $J A P$ analyzed data and wrote the paper, $B J D$ designed research and wrote the paper, $B L$ designed research, performed research, analyzed data, and wrote the paper, SES designed research, performed research, analyzed data, and wrote the paper

\section{Conflict of Interest Statement}

SM, KSC, SAM, PM, ASC, and JAP are all paid employees of Gilead Sciences Inc. BJL was a paid employee of Gilead Sciences Inc. when this research was performed. BJD is a paid consultant for Gilead Sciences Inc. SES receives research funding from Gilead Sciences Inc.

\section{REFERENCES}

1. Chiorazzi N, Rai KR, Ferrarini M. Chronic lymphocytic leukemia. N Engl J Med. 2005; 352(8): 804-15.

2. Weistner A. Emerging role of kinase-targeted strategies in chronic lymphocytic leukemia. Hematology Am Soc Hematol Educ Program. 2012; 120 (24): 4684-91.

3. Burger JA, Ghia P, Rosenwald A, Caligaris-Cappio F, The microenvironment in mature B-cell malignancies: a target for new treatment strategies. Blood. 2009; 114(16): 336775.

4. Audrito V,Vaisitti T, Serra S, Bologna C, Brusa D, Malavasi F, Deaglio S. Targeting the microenvironment in chronic lymphocytic leukemia offers novel therapeutic options. Cancer Lett. 2013; 328(1): 27-35.

5. Herman SE, Gordon AL, Wagner AJ, Heerema NA, Zhao W, Flynn JM, Jones J, Andritsos L, Puri KD, Lannutti BJ, Giese NA, Zhang X, Wei L, Byrd JC, Johnson AJ, Phosphatidylinositol 3-kinase-delta inhibitor CAL-101 shows promising preclinical activity in chronic lymphocytic leukemia by antagonizing intrinsic and extrinsic cellular survival signals. Blood. 2010; 116(12): 2078-88.

6 Herman SE, Gordon AL, Hertlein E, Ramanunni A, Zhang X, Jaglowski S, Flynn J, Jones J, Blum KA, Buggy JJ, Hamdy A, Johnson AJ, Byrd JC. Bruton tyrosine kinase represents a promising therapeutic target for treatment of chronic lymphocytic leukemia and is effectively targeted by PCI-32765. Blood. 2011; 117(23): 6287-96.

7. Quiroga MP, Balakrishnan K, Kurtova AV, Sivina M, Keating MJ, Wierda WG, Gandhi V, Burger JA, B-cell 
antigen receptor signaling enhances chronic lymphocytic leukemia cell migration and survival: specific targeting with a novel spleen tyrosine kinase inhibitor, R406. Blood. 2009; 114(5): 1029-37.

8. Buchner M, Baer C, Prinz G, Dierks C, Burger M, Zenz T, Stilgenbauer S, Jumaa H, Veelken H, Zirlik K, Spleen tyrosine kinase inhibition prevents chemokine- and integrinmediated stromal protective effects in chronic lymphocytic leukemia. Blood. 2010. 115(22); 4497-506.

9. Byrd JC, Furman RR, Coutre SE, Flinn IW, Burger JA, Blum KA, Grant B, Sharman JP, Coleman M, Wierda WG, Jones JA, Zhao W, Heerema NA, Johnson AJ, Sukbuntherng J, Chang BY, Clow F, Hedrick E, Buggy JJ, James DF, O'Brien S. Targeting BTK with ibrutinib in relapsed chronic lymphocytic leukemia. N Engl J Med. 2013; 369 (1): 32-42.

10. Böttcher S, Ritgen M, Fischer K, Stilgenbauer S, Busch RM, Fingerle-Rowson G, Fink AM, Bühler A, Zenz T, Wenger MK, Mendila M, Wendtner CM, Eichhorst BF, Döhner H, Hallek MJ, Kneba M. Minimal residual disease quantification is an independent predictor of progressionfree and overall survival in chronic lymphocytic leukemia: a multivariate analysis from the randomized GCLLSG CLL8 trial. J Clin Oncol. 2012; 30 (9): 980-8.

11. Chou TC,Talalay P. Quantitative analysis of dose-effect relationships: the combined effects of multiple drugs or enzyme inhibitors. Adv Enzyme Regul. 1984; 22: 27-55.

12. Lee JJ., Kong M, Ayers GD, Lotan R, Interaction index and different methods for determining drug interaction in combination therapy. J Biopharm Stat. 2007; 17(3): 461-80.

13. Herishanu Y, Pérez-Galán P, Liu D, Biancotto A, Pittaluga S, Vire B, Gibellini F, Njuguna N, Lee E, Stennett L, Raghavachari N, Liu P, McCoy JP, Raffeld M, StetlerStevenson $\mathrm{M}$, et al. The lymph node microenvironment promotes B-cell receptor signaling, NF-kappaB activation, and tumor proliferation in chronic lymphocytic leukemia. Blood. 2011; 117(2): 563-74.

14. Panayiotidis $\mathrm{P}$, Jones D, Ganeshaguru K, Foroni L, Hoffbrand AV, Human bone marrow stromal cells prevent apoptosis and support the survival of chronic lymphocytic leukaemia cells in vitro. Br J Haematol. 1996; 92(1): 97103.

15. de la Fuente MT Casanova B, Moyano JV, Garcia-Gila M, Sanz L, Garcia-Marco J, Silva A, Garcia-Pardo A, Engagement of alpha4beta1 integrin by fibronectin induces in vitro resistance of B chronic lymphocytic leukemia cells to fludarabine. J Leukoc Biol. 2002; 71(3): 495-502.

16. Bottcher S, Ritgen M, Fischer K, Stilgenbauer S, Busch RM, Fingerle-Rowson G, Fink AM, Bühler A, Zenz T, Wenger MK, Mendila M, Wendtner CM, Eichhorst BF, Döhner H, Hallek MJ, Kneba M. Minimal residual disease quantification is an independent predictor of progressionfree and overall survival in chronic lymphocytic leukemia: a multivariate analysis from the randomized GCLLSG CLL8 trial. J Clin Oncol. 2012; 30(9): 980-8.
17. Woyach JA, Johnson AJ, Byrd JC. The B-cell receptor signaling pathway as a therapeutic target in CLL. Blood. 2012; 120(6): 1175-84.

18. Li HL, Davis W, Pure E. Suboptimal cross-linking of antigen receptor induces Syk-dependent activation of p70S6 kinase through protein kinase $\mathrm{C}$ and phosphoinositol 3-kinase. J Biol Chem. 1999; 274(14): 9812-20.

19. Lannutti BJ, Meadows SA, Herman SE, Kashishian A, Steiner B, Johnson AJ, Byrd JC, Tyner JW, Loriaux MM, Deininger M, Druker BJ, Puri KD, Ulrich RG, Giese NA, CAL-101, a p110delta selective phosphatidylinositol-3kinase inhibitor for the treatment of B-cell malignancies, inhibits PI3K signaling and cellular viability. Blood. 2011; 117(2): 591-4.

20. Gobessi S, Laurenti L, Longo PG, Carsetti L, Berno V, Sica S, Leone G, Efremov DG, Inhibition of constitutive and BCR-induced Syk activation downregulates Mcl-1 and induces apoptosis in chronic lymphocytic leukemia B cells. Leukemia. 2009; 23(4): 686-97.

21. Davids MS, Burger JA. Cell Trafficking in Chronic Lymphocytic Leukemia. Open J Hematol. 2012; 3(S1).

22. Ghia P, Strola G, Granziero L, Geuna M, Guida G, Sallusto F, Ruffing N, Montagna L, Piccoli P, Chilosi M, CaligarisCappio F, Chronic lymphocytic leukemia B cells are endowed with the capacity to attract CD4+, CD40L + T cells by producing CCL22. Eur J Immunol. 2002; 32(5): 140313.

23. Burgess $M$, Cheung $C$, Chambers L, Ravindranath $\mathrm{K}$, Minhas G, Knop L, Mollee P, McMillan NA, Gill D, CCL2 and CXCL2 enhance survival of primary chronic lymphocytic leukemia cells in vitro. Leuk Lymphoma. 2012; 53(10): 1988-98.

24. Burger JA, Tsukada N, Burger M, Zvaifler NJ, Dell'Aquila M, Kipps TJ. Blood-derived nurse-like cells protect chronic lymphocytic leukemia B cells from spontaneous apoptosis through stromal cell-derived factor-1. Blood. 2000; 96(8): 2655-63.

25. Hoellenriegel, J, Coffey GP, Sinha U, Pandey A, Sivina M, Ferrajoli A, Ravandi F, Wierda WG, O'Brien S, Keating MJ, Burger JA. Selective, novel spleen tyrosine kinase (Syk) inhibitors suppress chronic lymphocytic leukemia B-cell activation and migration. Leukemia. 2012; 26 (7): 1576-83. 